\title{
Metronomics for thymic carcinoma
}

Jacobo Muñoz del Toro, Patricia Cortez Castedo, Alfonso Cortés Salgado, M Eugenia Olmedo García and Pilar Garrido López

Medical Oncology Department, Hospital Universitario Ramón y Cajal, Ctra de Colmenar Viejo km 9, Madrid 28034, Spain

Correspondence to: Jacobo Muñoz del Toro. Email: jacobomdeltoro@gmail.com

\section{Abstract}

Although thymomas are the most frequent primary tumours of the anterior mediastinum, thymic carcinoma is very infrequent and more aggressive. Combination chemotherapy is the first-line treatment for the advanced stages, but because of the lack of evidence from randomised trials, the management of the successive lines is a challenging field. We report a partial radiological response in the seventh line of a thymic carcinoma stage IV with an oral regimen.

Keywords: metronomics, recurrent thymic carcinoma, treatment

Published: $18 / 12 / 2014$

Received: 21/08/2014

ecancer 2014, 8:494 DOI: 10.3332/ecancer.2014.494

Copyright: $\odot$ the authors; licensee ecancermedicalscience. This is an Open Access article distributed under the terms of the Creative Commons Attribution License (http://creativecommons.org/licenses/by/3.0), which permits unrestricted use, distribution, and reproduction in any medium, provided the original work is properly cited. 


\section{Introduction}

Thymic epithelial tumours are divided into two categories, thymomas and thymic carcinomas. Although thymic tumours are rare (0.2-1.5\% of all malignancies; 1.3 cases per million population in the US [1]), thymomas are the most frequent primary tumours of the anterior mediastinum [2]. On the other hand, thymic carcinoma is responsible for less than $1 \%$ of thymic neoplasms. It is more aggressive than thymoma and often metastasizes [3-4]. The differential diagnosis between thymoma and thymic carcinoma is complex and mainly based on histological and immunohistochemical differences [5]. We report the case of a patient with an initial stage IVA Masaoka-Koga staging system thymic carcinoma lasting 11 years who achieved a partial response by using a metronomic approach in his seventh line of treatment.

\section{Case report}

The patient was a 41-year-old male with no relevant medical history who in October 2001 was diagnosed by biopsy of a stage IVA (pleural involvement) thymic carcinoma. The patient received first-line chemotherapy in other hospital. The selected regimen was cisplatin, adriamycin, and cyclophosphamide (CAP) followed by etoposide, ifosfamide, and cisplatin (VIP) completing six cycles, achieving a partial response and passing to monitoring. After a progression-free interval of 4 years, a relapse was diagnosed including pulmonary metastasis together with positivity for somatostatin receptor scintigraphy. The patient was staged as stage IVB, and for the next 7 years, the patient received several treatment lines successively, including lanreotide and steroids, gefitinib, paclitaxel, carboplatin, etoposide, sunitinib as well as palliative radiotherapy. The patient achieved prolonged stabilizations as best response lasting less than 1 year each.

On February 2012, the patient was admitted to our hospital, where a new line of treatment was offered. The regimen included continuous oral cyclophosphamide (50 mg/day) and prednisone (100 mg per day) plus monthly intramuscular lanreotide at a dose of $60 \mathrm{mg}$. After two cycles, a partial response was visible on the CT scan (Figure 1). The patient continued with the same regimen except cyclophosphamide that had to be abandoned after the fourth cycle due to a progressive deterioration of the renal function. Additionally, the patient had anaemia grade 2 and fatigue grade 1 as more relevant toxicity. With this schema, the patient showed a clinically significant benefit and radiological partial response for more than 1 year. Finally, a pulmonary progression was detected, and the patient died in November 2013.
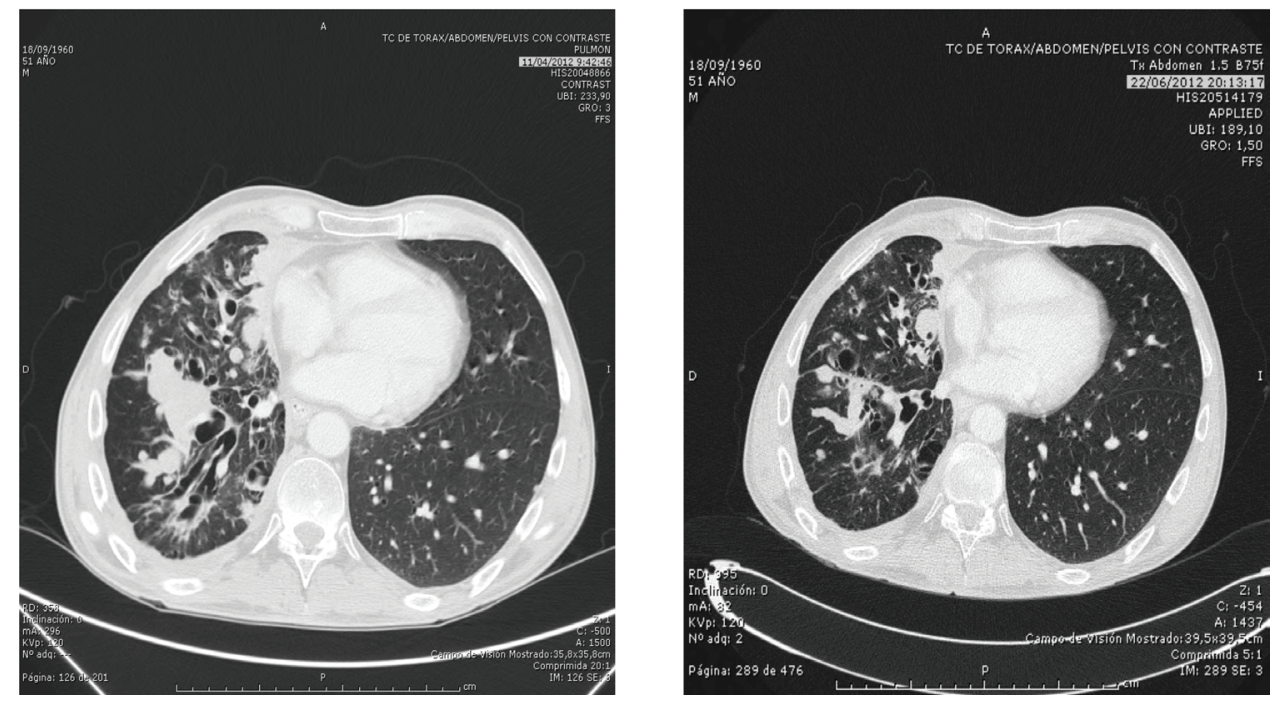

Figure 1. The response after metronomic treatment. 


\section{Discussion}

The behaviour of thymic carcinomas is more aggressive than thymomas, with a 5-year overall survival around 40\% [3-4]. The recommended first-line treatment for advanced stages is combination chemotherapy, usually platinum-based schemes, with the most common being CAP, ADOC (cisplatin, doxorubicin, vincristine, and cyclophosphamide) and carboplatin-paclitaxel regimes, and an overall response rate ranging between $30 \%$ and $50 \%$ and a median progression-free interval of approximately 6 months [6-8]. Unfortunately, the evidence of the benefit of treatment with successive lines is scarce, mainly based on studies with a few patients or an isolated case report. There are small amounts of data published using chemotherapeutic drugs (ifosfamide, cisplatin, etoposide, irinotecan, or pemetrexed) and other agents (octreotide with or without prednisone, belinostat, erlotinib, gefinitib, imatinib, sacaratinib, sorafenib, sunitinib, or cetuximab) [9-11].

Metronomic treatment is the chronic administration of chemotherapy at low, minimally toxic doses on a frequent schedule of administration, with no prolonged drug-free breaks [12]. Although the mechanism of action of metronomic chemotherapy is not fully defined, antiangiogenic and antitumor effects—by restoration of the immune system—have been postulated [12, 14]. In this particular area, Kivrak et al [13] recently published a case report showing a complete response in a patient with recurrent thymic carcinoma treated with cyclophosphamide and etoposide in a metronomic strategy. Moreover, André et al proposed a new strategy based on the combination of metronomic chemotherapy and drug reposition named metronomics $[15,16]$. In our patient, this metronomics approach was successful, offering to the patient a relevant clinical benefit with low toxicity and a partial radiological response in his seventh line of treatment, being the only objective response since 2005. In our experience, the metronomics approach deserves further investigation in this subset of patients.

\section{Conclusions}

The interest of this case lies in the response to the therapy used, taking into account that it was a thymic carcinoma with an evolution of 11 years, and that all components of the scheme had already been used in previous lines, but not using a metronomic approach. To our knowledge, there are no published studies using this combination in such as advanced line. The good tolerability and the advantages of the oral administration suggest that this combination should be further analysed.

\section{Conflicts of interest}

The authors have no conflicts of interest to declare.

\section{References}

1. Engels EA (2010) Epidemiology of thymoma and associated malignancies $J$ Thorac Oncol 5 260-5 DOI: 10.1097/JTO.0b013e318 1f1f62d

2. Muller-Hermelink HK et al (1993) The pathological basis of thymoma-associated myasthenia gravis Ann NY Acad Sci 681 56-65 DOI: 10.1111/j.1749-6632.1993.tb22869.x PMID: $\underline{7689313}$

3. Strollo DC, Rosado de Christenson ML and Jett JK (1997) Primary mediastinal tumors Part 1: tumors of the anterior mediastinum Chest 112 511-22 DOI: $10.1378 /$ chest.112.2.511 PMID: $\underline{9266892}$

4. Eng TY et al (2004) Thymic carcinoma: a state of the art review Int J Radiat Oncol Biol Phys 59 654-64 DOI: 10.1016/j.jirobp. 2003.11.021 PMID: 15183468

5. Marx A et al (2011) Thymic carcinoma: is it a separate entity? From molecular to clinical evidence Thorac Surg Clin 21 25-31 v-vi DOI: 10.1016/j.thorsurg.2010.08.010 
6. Loehrer PJ Sr et al (1994) Cisplatin plus doxorubicin plus cyclophosphamide in metastatic or recurrent thymoma: final results of an intergroup trial. The Eastern Cooperative Oncology Group, Southern Oncology Group, Southwest Oncology Group and Southeastern Cancer Study Group J Clin Oncol 12 1164-8 PMID: 8201378

7. Fornasiero A et al (1991) Chemotherapy for invasive thymoma. A 13 year experience Cancer 68 30-3 DOI: 10.1002/1097-0142(19910701)68:1\&lt;30::AID-CNCR2820680106\&gt;3.0.CO;2-4 PMID: 2049749

8. Okuma $Y$ et al (2014) Key components of chemotherapy for thymic malignancies: a systematic review and pooled analysis for anthracycline-, carboplatin- or cisplatin-based chemotherapy J Cancer Res Clin Oncol Aug 22 DOI: 10.1007/s00432-014$\underline{1800-6}$

9. Koppitz H et al (2012) State-of-the-art classification and multimodality treatment of malignant thymoma Cancer Treatment 38 540-8 DOI: 10.1016/j.ctrv.2011.11.010

10. Kelly RJ et al (2011) Thymic malignancies: from clinical management to targeted therapies J Clin Oncol 29 2840-7 DOI: 10.1200/ JCO.2011.36.0487

11. Ettinger DS et al (2013) Thymomas and thymic carcinomas: Clinical Practice Guidelines in Oncology J Natl Compr Canc Netw 11(5) 562-76 PMID: $\underline{23667206}$

12. Pasquier E, Kavallaris $M$ and André N (2010) Metronomic chemotherapy: new rationale for new directions Nat Rev Clin Oncol 7 455-65 DOI: 10.1038/nrclinonc.2010.82 PMID: 20531380

13. Kivrak D et al (2014) Metronomic maintenance chemotherapy in patients presenting with paraneoplastic autoimmune hepatitis with recurrent thymic carcinoma J Oncol Pharm Pract Sep 16 DOI: $10.1177 / 1078155214551318$

14. Kerbel RS and Kamen BA (2004) The anti-angiogenic basis of metronomic chemotherapy Nat Rev Cancer 4 23-36 DOI: $10.1038 /$ $\underline{\text { nrc1369 }}$

15. Ashburn TT and Thor KB (2004) Drug repositioning: identifying and developing new uses for existing drugs Nat Rev Drug Discov 3 673-83 DOI: 10.1038/nrd1468 PMID: 15286734

16. André $\mathrm{N}$ et al (2013) Has the time come for metronomics in low-income and middle-income countries? Lancet Oncol 14(6) e239-48 DOI: 10.1016/S1470-2045(13)70056-1 PMID: 23639324 